\title{
ARTICLE OPEN \\ Systematic beyond-DFT study of binary transition metal oxides
}

\author{
Subhasish Mandal $\mathbb{D}^{1 *}{ }^{*}$, Kristjan Haule ${ }^{1}$, Karin M. Rabe ${ }^{1}$ and David Vanderbilt (iD ${ }^{1}$
}

Various methods going beyond density functional theory (DFT), such as DFT+U, hybrid functionals, meta-GGAs, GW, and DFTembedded dynamical mean field theory (eDMFT), have been developed to describe the electronic structure of correlated materials, but it is unclear how accurate these methods can be expected to be when applied to a given strongly correlated solid. It is thus of pressing interest to compare their accuracy as they apply to different categories of materials. Here we introduce a novel paradigm in which a chosen set of beyond-DFT methods is systematically and uniformly tested on a chosen class of materials. For a first application, we choose the target materials to be the binary transition metal oxides $\mathrm{FeO}, \mathrm{CoO}, \mathrm{MnO}$, and $\mathrm{NiO}$ in their antiferromagnetic phase and present a head-to-head comparison of spectral properties as computed using the various methods. We also compare with available experimental angle-resolved photoemission spectroscopy (ARPES), inverse-photoemission spectroscopy, and with optical absorption. For the class of compounds studied here, we find that both B3LYP and eDMFT reproduce the experiments quite well, with eDMFT doing best, in particular when comparing with the ARPES data.

npj Computational Materials (2019)5:115; https://doi.org/10.1038/s41524-019-0251-7

\section{INTRODUCTION}

Future technologies depend on new materials with tailored, enhanced, and/or novel functionalities. Strongly correlated materials exhibit rich physics that offers unique opportunities in this regard, particularly in their magnetic, optical, and transport properties. ${ }^{1}$ While progress in computational materials design has greatly accelerated the process of identifying and realizing materials with moderate correlations, significant challenges remain for the quantitative, and in some cases even qualitative, computational prediction of the properties of interest of strongly correlated materials. Furthermore, accurate computation of excited-state properties even in moderately correlated materials is beyond the scope of density functional theory (DFT), ${ }^{2-4}$ and in strongly correlated materials, it is well known that DFT can even fail correctly to predict whether a system is a metal or an insulator. The existing materials databases (https://materialsproject.org), constructed in the spirit of the Materials Genome Initiative (https://www.mgi.gov), are built almost exclusively by DFT engines and are thus very often making incorrect predictions in correlated materials. There is thus a longstanding interest in developing perturbative, stochastic, and hybrid functional approaches, referred to as "beyond-DFT" methods, to treat strongly correlated and excited-state properties. ${ }^{5-8}$

In strongly correlated materials, one reason for the failure of DFT is the delocalization or self-interaction error, ${ }^{9,10}$ which can be partially fixed by adding a Hubbard $U$ in the DFT $+U$ approach. ${ }^{11}$ This method recovers the insulating state in many materials that are incorrectly predicted to be metallic in DFT. ${ }^{11-13}$ Moreover, DFT and $\mathrm{DFT}+\mathrm{U}$ methods give quite accurate results for structural parameters in most materials (https://materialsproject.org). ${ }^{14}$ However, unique determination of an appropriate value of $U$ for more general quantitative calculations has proved surprisingly problematic. Hybrid functionals, ${ }^{15,16}$ which also correct most of the self-interaction error by incorporating a certain fraction of exact exchange, significantly improve the descriptions of many $d$-electron systems. ${ }^{15-18}$ The fraction of exact exchange is treated as a tuning parameter, generally falling in the range $\sim 0.2-0.45$.

Many-body perturbation theory in the GW quasiparticle approximation $^{19}$ is a beyond-DFT method that was developed to better describe the quasiparticle excitations in solids, and results compare well with the experimental photoemission spectroscopy and inverse photoemission spectroscopy (PES/IPES) for many semiconducting and insulating systems with open $s$ and $p$ shells. ${ }^{2,20,21}$ Dynamical mean field theory (DMFT) ${ }^{3}$ gives an exact treatment of intrashell correlations local to a given ion. Originally developed for lattice models, its combination with DFT in the socalled DFT+DMFT method ${ }^{4}$ allows for a quantitative description of the electronic structure of strongly correlated materials such as Febased superconductors, ${ }^{22-25,25,26}$ Mott insulators, ${ }^{27}$ and heavy fermion systems $s^{28}$ without tuning parameters ${ }^{29,30}$ Furthermore, DFT+DMFT calculations of the spectral function have been instrumental for the understanding of PES/IPES for a variety of strongly correlated systems.

The beyond-DFT methods vary greatly in their suitability for different classes of correlated materials ${ }^{5-7}$. In addition, they are considerably more computationally intensive than DFT or DFT $+\mathrm{U}$. Especially in the context of high-throughput studies, ${ }^{5,6}$ this means that there is a pressing need for a systematic way to choose, for any given material, the computational method that will give physically accurate results without unnecessary computation. Development of this capability requires that the performance of various beyond-DFT methods be systematically and uniformly tested on a diverse training set of strongly correlated materials that are experimentally well characterized. ${ }^{5-7}$

Binary transition metal oxides (TMOs) are among the most thoroughly studied strongly correlated materials ${ }^{11-13,15,31-43}$ and thus are a natural starting point for generation of the training set. They include a number of wide-gap insulators predicted to be metallic in the conventional density functional formalism (DFT). ${ }^{13,44}$ Those that contain early and late transition metals are usually categorized as "Mott" and "charge-transfer" insulators in

\footnotetext{
${ }^{1}$ Department of Physics and Astronomy, Rutgers University, Piscataway, NJ, USA. *email: sm2159@physics.rutgers.edu
} 
the "Zaanen-Sawatzky-Allen" scheme ${ }^{45}$ and are insulating both above and below the Neel ordering temperature, with strongly localized $3 d$ magnetic moments. These localized magnetic moments, originating in the $3 d$ TM states, hybridize with more itinerant $4 s$ and oxygen $2 p$ states, resulting in competition between localization and itinerancy. ${ }^{32,33}$ Another reason that binary TMOs are ideal for the training set is that their crystal structures are very simple. ${ }^{15}$ In the paramagnetic phase, they crystallize in the rock-salt $(\mathrm{Fm} 3 \mathrm{~m})$ structure; at lower temperatures, antiferromagnetic ordering (AFM $\mathrm{II})^{46}$ results in a rhombohedral $(\mathrm{R} 3 \mathrm{~m})$ structure, with two transition metal ions in the unit cell.

In this paper, we systematically and uniformly test various beyond-DFT methods on the set of binary TMOs $\mathrm{MnO}, \mathrm{FeO}, \mathrm{CoO}$, and $\mathrm{NiO}$, allowing a head-to-head comparison between the various methods with experimental photoemission and inverse photoemission measurements. The methods included in the study are generalized gradient approximation (GGA) with the Perdew-Burke-Ernzerhof (PBE) functional, ${ }^{47} \mathrm{GGA}+\mathrm{U}$ with the Anisimov and Lichtenstein formalism, ${ }^{48,49}$ meta-GGA with the modified Becke-Johnson (mBJ) potential, ${ }^{50}$ all-electron $\mathrm{GW}_{0}{ }^{51}$ in the Hedin formalism, hybrid functionals with B3LYP, ${ }^{52}$ and DFT +DMFT in the DFT+Embedded DMFT (eDMFT) formalism (http:// hauleweb.rutgers.edu/tutorials). ${ }^{53,54}$ In addition, optical properties are computed with B3LYP and eDMFT and compared with the available experiments. We expect to expand the training set of materials dramatically in future work, with the eventual goal of

(a)

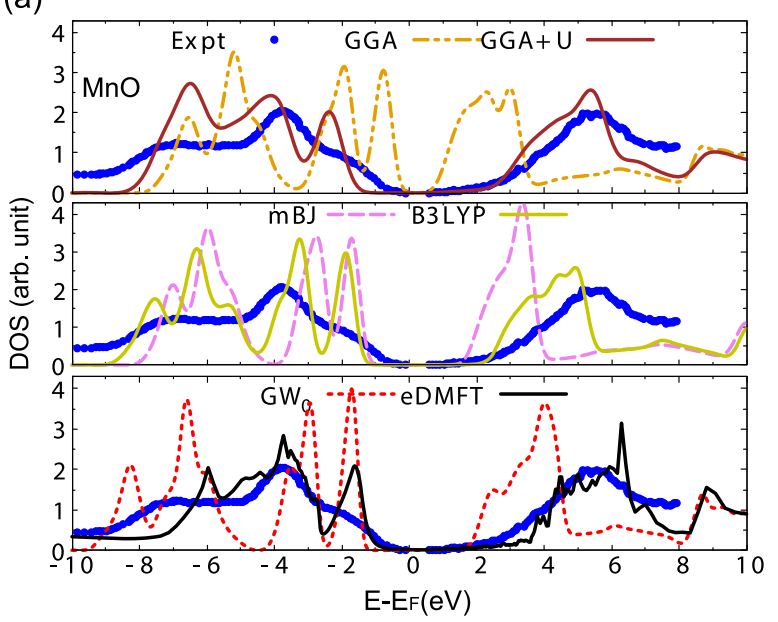

(b)

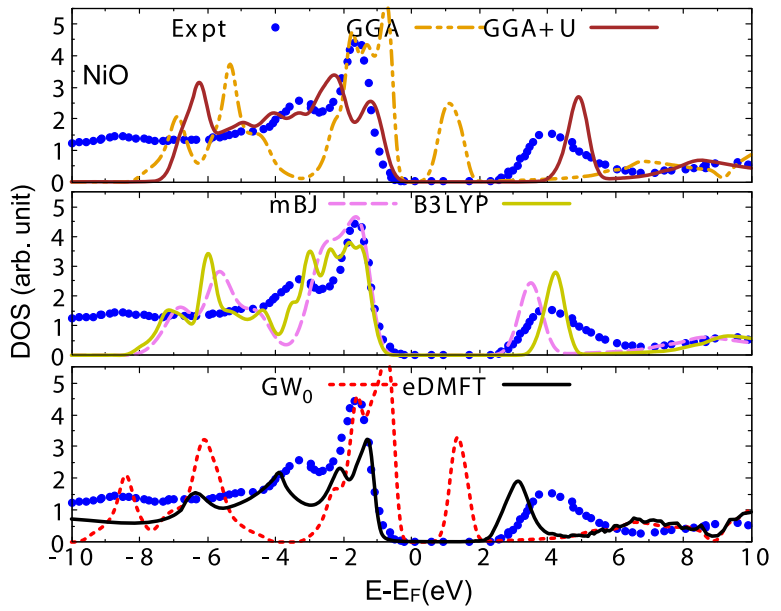

constructing a database in which a chosen set of DFT and beyondDFT methods are systematically applied to an increasingly wide range of materials. Such a database holds great promise to enhance the power of computational materials design and discovery.

The calculations are performed for $\mathrm{MnO}, \mathrm{FeO}, \mathrm{CoO}$, and $\mathrm{NiO}$ in the rocksalt structure ${ }^{15}$ with experimentally reported lattice parameters $a=4.445,{ }^{55} 4.334,{ }^{56} 4.254,{ }^{57}$ and $4.171 \AA^{58}$ respectively. Magnetic ordering is taken as AFM II along [111], ${ }^{46}$ which leads to an R3m space group symmetry with two TM ions in the unit cell. Calculations for the paramagnetic state will be considered separately in a future publication (ref. ${ }^{59}$ ).

\section{RESULTS}

Density of states (DOS)

DOS is shown in Fig. 1 as obtained by GGA, GGA+U, mBJ, GW, B3LYP, and eDMFT methods. From the computed DOS, we see directly whether a material is predicted to be a metal or insulator with a given method. Another important point of comparison is the splitting between the peak at the top of the valence band and the lowest peak in the conduction band, which is quantified experimentally as the PES/IPES gap. For comparison, in each subplot, we include the experimental photoemission (PES) and inverse photoemission (IPES) for $\mathrm{MnO}, \mathrm{NiO}, \mathrm{CoO}$, and $\mathrm{FeO}$ from refs. ${ }^{40-43}$. Since the experimental PES and IPES spectra are

(c)

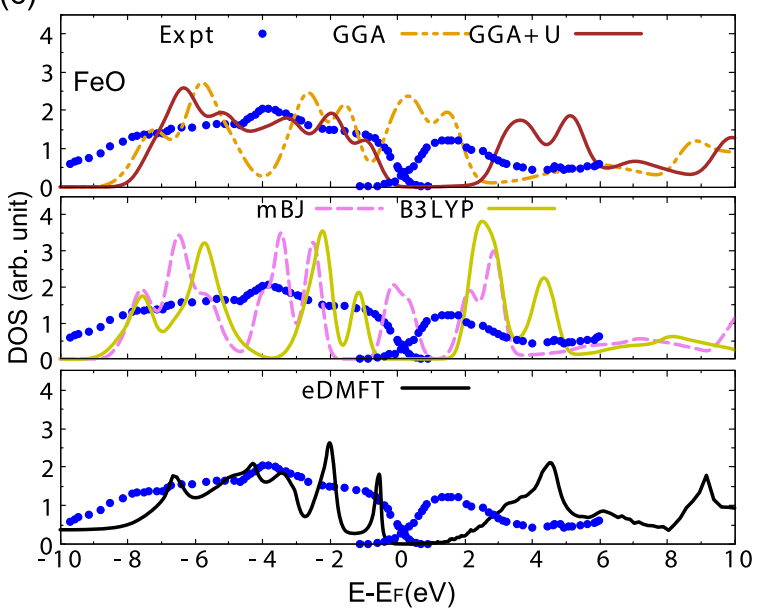

(d)

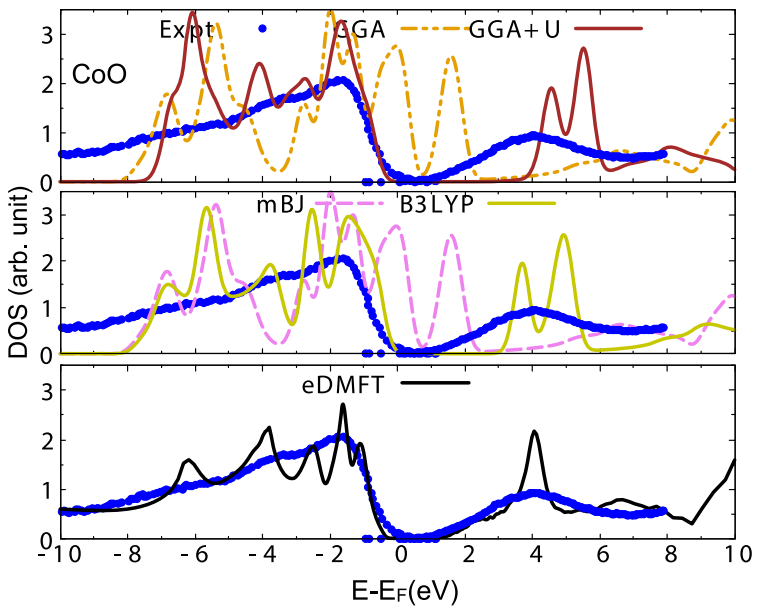

Fig. 1 Comparison of total density of states (DOS) in various DFT and beyond-DFT methods: Total DOS (states/eV) as computed in GGA, $\mathrm{GGA}+\mathrm{U}, \mathrm{mBJ}, \mathrm{B} 3 \mathrm{LYP}, \mathrm{GW}_{0}$, and eDMFT for a MnO, b NiO, c FeO, and d CoO. Blue dots indicate photoemission and inverse photoemission data in arbitrary unit as obtained from refs. ${ }^{40-43}$ for $\mathrm{MnO}, \mathrm{NiO}, \mathrm{CoO}$, and $\mathrm{FeO}$, respectively. 
reported in arbitrary units, we have arbitrarily re-scaled them to fit in the range of the computed DOS. Note also that we compare them to the total DOS, which is the best choice for off-resonance spectra of PES/IPES, and we expect to see correct peak positions and similar intensities, although the precise intensity of each peak is not expected to be achieved here, as that would require one to compute the matrix elements for PES/IPES processes. Finally, let us mention that experimentally PES is expected to be more precise than IPES, and the latter generally has larger broadening due to experimental resolution. For this reason, we avoid quoting a single number for the band gap, although it is common to do so in the literature.

For $\mathrm{MnO}$, all six methods predict that the AFM systems are insulating. As expected, GGA substantially underestimates the experimental PES/IPES gap. With the value of $U=6.04 \mathrm{eV}^{15} \mathrm{GGA}$ $+U$ predicts the experimental PES/IPES gap accurately. Similarly, the $\mathrm{mBJ}, \mathrm{B} 3 \mathrm{LYP}$, and $\mathrm{GW}_{0}$ methods also show improved agreement for the $\mathrm{MnO}$ peak splitting relative to GGA, without using any material-specific tuning parameters. While it is slightly underestimated by B3LYP, the agreement with the experimental PES/IPES of MnO is best with the eDMFT method.

For $\mathrm{NiO}$, we find similar results. All methods predict an insulating character, with GGA underestimating the experimental PES/IPES gap. $G_{0}$ does not improve the spectrum much from its GGA shape. On the other hand, the meta-GGA with the $\mathrm{mBJ}$ functional performs remarkably well. As was the case for $\mathrm{MnO}$, both B3LYP and eDMFT predict the experimental PES/IPES spectra very well (Fig. 1b), with a slightly better match in B3LYP.
$\mathrm{FeO}$ and $\mathrm{CoO}$ are the most challenging cases, as the regular GGA predicts them to be metallic. Using $U=5.91$ and $6.88 \mathrm{eV}$ for $\mathrm{FeO}$ and $\mathrm{CoO}$, respectively, ${ }^{15}$ both the systems recover insulating phase. $\mathrm{mBJ}$ predicts metallic solutions for $\mathrm{FeO}$ and $\mathrm{CoO}$, similar to GGA (Fig. 1c, d). It is noteworthy to mention that, depending on the starting local density matrix, the basis set, the crystal structures (whether experimental or optimized), and the software used, multiple solutions are often found in the literature for $\mathrm{FeO}$ and $\mathrm{CoO}$ in $\mathrm{DFT}+\mathrm{U}$ and $\mathrm{mBJ}$ methods. ${ }^{7,13} \mathrm{GW}_{0}$ on top of local density approximation (LDA) also predicts both $\mathrm{FeO}$ and $\mathrm{CoO}$ to be metallic (not shown). Both B3LYP and eDMFT show a very good agreement with the PES/IPES (Fig. 1) for all four TMOs, except for $\mathrm{FeO}$. As was emphasized in the literature, ${ }^{60} \mathrm{FeO}$ crystals tend to be non-stoichiometric, which is the likely cause of inadequate IPES spectrum. It would be desirable to repeat this experiment on more stoichiometric single crystals. Overall, eDMFT performs best for describing the peak positions. In particular, for $\mathrm{MnO}$ and $\mathrm{CoO}$ it predicts the gradual increase of the conduction band intensity for the unoccupied states in good agreement with IPES.

\section{Spectral function}

From the above discussion, it is clear that only B3LYP and eDMFT can consistently reproduce the experimental PES/IPES peak positions without using a material-specific tuning parameter for all four TMOs. Hence, we discuss spectral functions as computed by these two methods, shown in Fig. 2, which reproduce and go beyond the discussion of the DOS. For example, we see again that
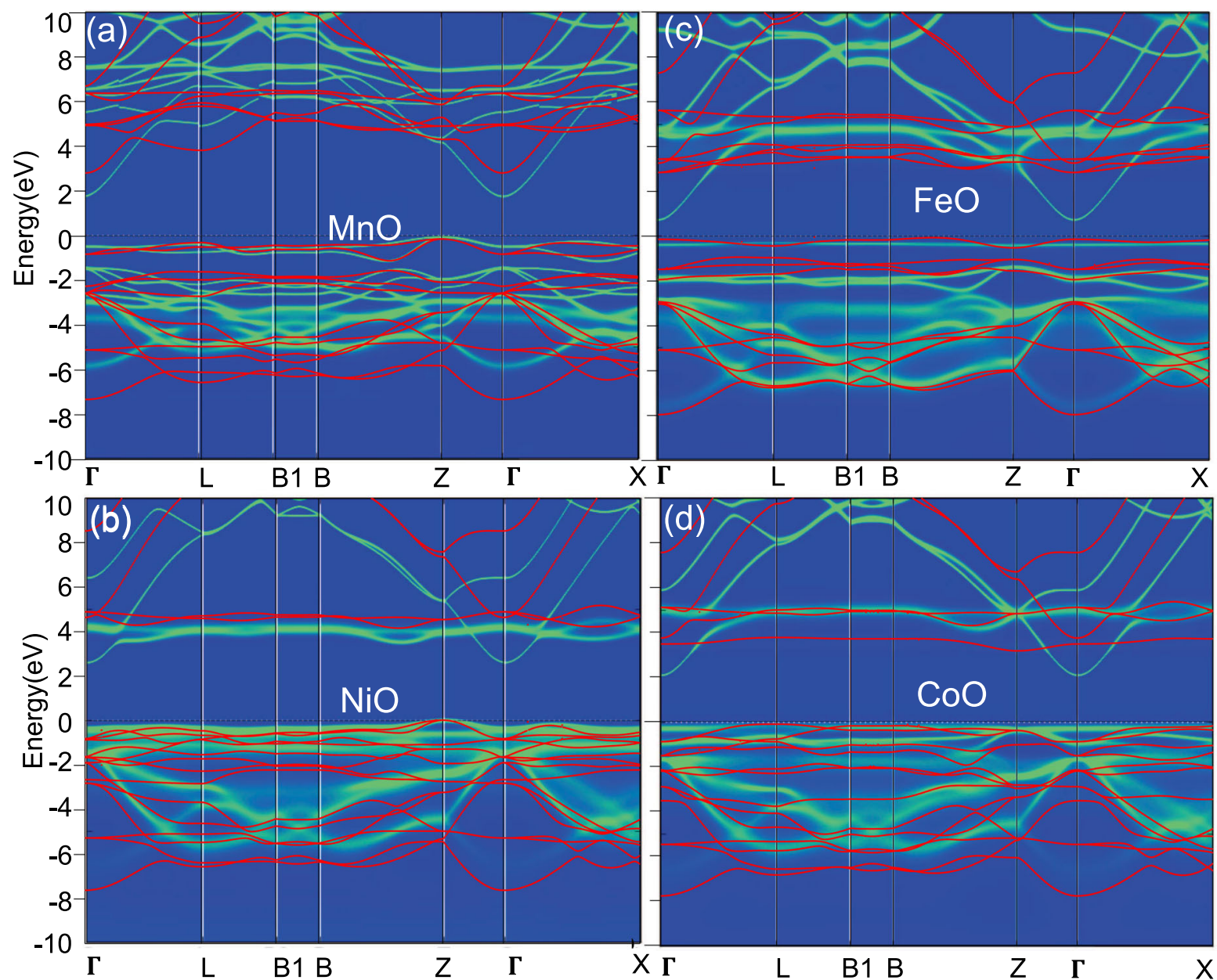

Fig. 2 Comparison of band structures: Band structures and spectral functions as computed in B3LYP (red) and eDMFT (green) are compared for a $\mathrm{MnO}, \mathbf{b} \mathrm{NiO}, \mathbf{c} \mathrm{FeO}$, and $\mathbf{d} \mathrm{CoO}$. 


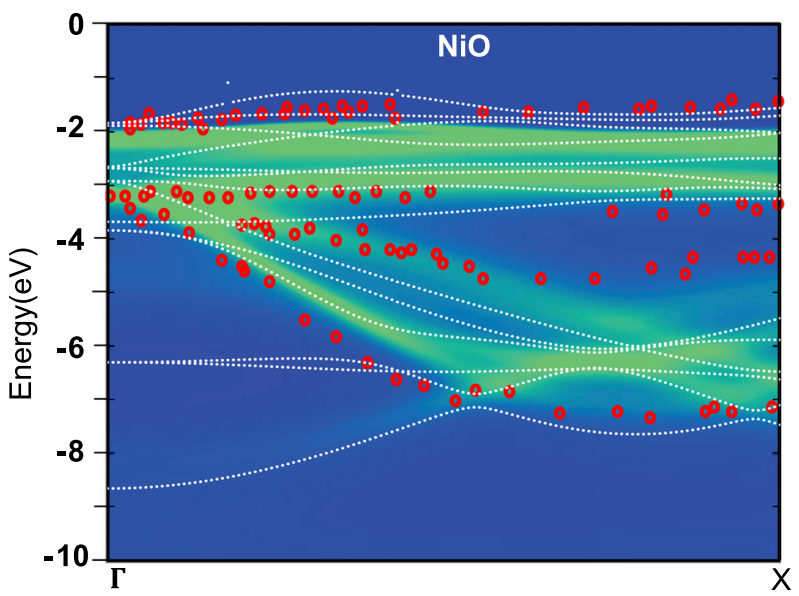

Fig. 3 Comparison of band structures with experiment: Experimental ARPES (in red dots) by Shen et al. are compared with spectral function as computed with eDMFT (green) and band structure with B3LYP (in white lines) for NiO. Experimental data are reproduced from ref. ${ }^{39}$.

splitting between occupied and unoccupied flat $3 d$ bands (previously discussed for the DOS) is larger in eDMFT than in B3LYP for $\mathrm{MnO}, \mathrm{CoO}$, and $\mathrm{FeO}$, while it is slightly smaller in $\mathrm{NiO}$. From the spectral function, we also learn about the dispersion of the key bands near the Fermi level. In general, we expect B3LYP to show more bands than eDMFT, as all spectral weight in static theories, like B3LYP, needs to come from sharp band excitations, while in eDMFT, part of the spectrum is incoherent and is redistributed as diffuse weight over a large energy range. We showed before (Fig. 1) that, except for NiO, eDMFT peak positions in the DOS are in slightly better agreement with PES/IPES, hence we expect the spectral functions of eDMFT are likely a better prediction for angle-resolved inverse photoemission as well. We compare eDMFT spectral functions (green) and B3LYP band structure (white) for $\mathrm{NiO}$ with the available angle-resolved photoemission spectroscopy (ARPES), measured by Shen et al. ${ }^{39}$ (Fig. 3). The ARPES data in $\mathrm{NiO}$ were taken in the AFM phase, consistent with our theoretical calculation. The vertical energy axes are shifted to match the experimental bands at the $\Gamma$ point near $\sim-2 \mathrm{eV}$, as the position of the chemical potential inside the gap is arbitrary in theory, and determined by the impurity concentration in the experiment.

Within eDMFT, the $4 s$ dispersing state appears as the first conduction band at the $\Gamma$ point in all four TMOs and gives a direct gap to be at the $\Gamma$ point. In B3LYP, this state similarly appears in $\mathrm{MnO}$ as the first conduction excitation but is shifted much higher in all other TMOs. The precise position of this state is hard to determine from currently available experimental data. As eDMFT does not treat this $4 s$ state as correlated, its position is not improved from its LDA description, and it might be too low in some TMOs. However, IPES does show a very gradual increase in the intensity in $\mathrm{MnO}$ and $\mathrm{CoO}$, due to the presence of this $4 \mathrm{~s}$ state, which agrees with eDMFT. Note that in $\mathrm{NiO}$ this $4 s$ state is shifted upwards compared to the flat $3 d$ states in eDMFT, and experimentally IPES in $\mathrm{NiO}$ does show a more abrupt and narrower unoccupied peak (Fig. 1). It is therefore tempting to speculate that the first conduction state in all four TMOs is such a dispersive $4 s$ band at the $\Gamma$ point, as predicted by eDMFT.

For $\mathrm{FeO}$ and $\mathrm{CoO}$, another prominent feature of the eDMFT spectral function is a very flat band just below the Fermi level. This flat band gives rise to a sharp peak in the occupied DOS near $E_{\mathrm{F}}$. Such a flat band is also observed in a GW computation done on top of hybrid functional ${ }^{61}$ but is not found in regular DFT $+U$ or hybrid approaches. We notice the similar flat band in the experimental APRES spectrum for $\mathrm{CoO}$ in the paramagnetic phase. ${ }^{59}$ For $\mathrm{FeO}$, we are not aware of any experimental ARPES data to compare with.

For $\mathrm{MnO}$, the eDMFT spectral function is much sharper than in other TMOs. This is due to the fact that the entire fluctuating moment orders in $\mathrm{MnO}$, which makes the system more mean field-like and less correlated. Unfortunately, there are no experimental data available for $\mathrm{MnO}$ to compare with.

In Fig. 3, we compare the computed spectral functions with experimental ARPES data for $\mathrm{NiO}$, which are the only one of our four materials for which such data are available in the AFM phase. We see that the experimental ARPES data match quite well with the eDMFT-predicted spectral function, while the B3LYP bands do not show many similarities with the experiment. In particular, at the $\Gamma$ point, experiment resolves only two peaks, with one flat state around $-2 \mathrm{eV}$ and another degenerate state around $-3 \mathrm{eV}$, in which two branches disperse downward, and one remains mostly flat toward the $X$ point. This is all in agreement with eDMFT, while B3LYP shows a very different pattern of degeneracy at the $\Gamma$ point, not matching ARPES. Moreover, B3LYP predicts several extra bands near the $\Gamma$ point, in particular near $-6.5 \mathrm{eV}$, which did not show up in ARPES and are absent in eDMFT. An additional extremely weak spectrum was observed for the uppermost valence band (not shown here), which was only noticed for selected photon energies and certain emission angles in the ARPES experiment. ${ }^{38,39}$ The uncertainties of this spectrum were discussed in refs. ${ }^{38,39}$. Finally, for an even better match of eDMFT spectrum and ARPES, one would need to shift the dispersive oxygen $p$ states slightly lower compared to the flat, mostly $3 d$ state at $-2 \mathrm{eV}$. This deficiency of LDA+eDMFT is known and is inherited from LDA, which tends to place oxygen $2 p$ states slightly close to the Fermi level.

\section{Optical absorption}

Finally, we present our results for the optical absorption, which measures the vertical (zero momentum transfer) transitions between the single-particle states, computed by B3LYP and eDMFT. In Fig. 4, we compare the absorption coefficient from the reflectivity measurements. For $\mathrm{MnO}$, the optical absorption is extracted from figure in Röld et al., ${ }^{61}$ where the measurements by Ksendzov et al. ${ }^{62}$ were reproduced. The original data for $\mathrm{MnO}$ are not currently accessible. For $\mathrm{NiO}$ and $\mathrm{CoO}$, the experimental absorption coefficients are extracted from Powell et al., ${ }^{63}$ which were obtained from the measured reflectivity spectra. Reliable reflectivity spectra performed on stoichiometric $\mathrm{FeO}$ are unavailable.

We observe various important points in Fig. 4. First, within eDMFT and B3LYP, the onset of the optical absorption is gradual in $\mathrm{MnO}, \mathrm{FeO}$, and $\mathrm{CoO}$, while it is much more sudden in $\mathrm{NiO}$. This gradual onset in eDMFT is due to the presence of a dispersive $4 \mathrm{~s}$ band around the $\Gamma$ point as discussed above.

Second, the peak positions in the experimental absorption and those computed by eDMFT agree very well. For example, the first peak for $\mathrm{NiO}$ in eDMFT is at $4.6 \mathrm{eV}$ while in the experiment it is at $4.9 \mathrm{eV}$. The overall shapes and magnitudes match very well in $\mathrm{NiO}$. Similarly, for $\mathrm{MnO}$, the overall shapes of eDMFT and experiment match very well. However, the first shoulder of intensity between 2.5 and $4 \mathrm{eV}$ seems to be missing in the data of Ksendzov et al., ${ }^{62}$ which seems somewhat inconsistent with the IPES in Fig. 1, where a slow increase of the intensity is noticed. To clarify the correct placement of the $\mathrm{Mn} 4 \mathrm{~s}$ states, it would be desirable to acquire new spectra for MnO obtained with modern techniques.

For $\mathrm{CoO}$, the eDMFT peak positions are similar to the experiment, but the overall match is not so good. B3LYP, which does not match well in $\mathrm{MnO}$ and $\mathrm{NiO}$ (the intensity is too small and peaks do not align), seems superior in $\mathrm{CoO}$, with an overall good match and a correct gap. 

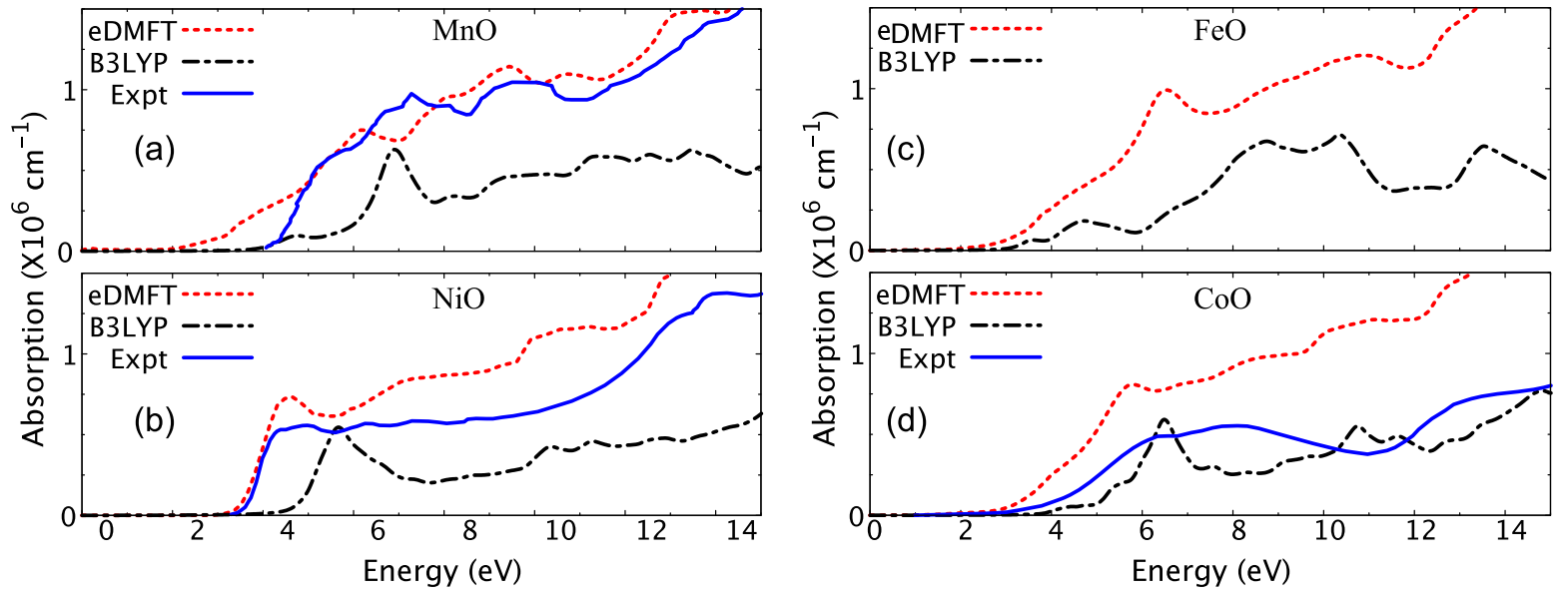

Fig. 4 Comparison of optical absorption coefficients: eDMFT and B3LYP computed optical absorption coefficients (in dashed lines) for all four TMOs are compared with available experiments (in solid lines). Experimental data are directly obtained from Powell et al. ${ }^{63}$ for CoO and NiO and from Rödl et al. ${ }^{61}$ for $\mathrm{MnO}$, which was used to compare with the experimental reflectivity by Ksendzov et al. ${ }^{62}$.

\section{DISCUSSION}

We find that only B3LYP and eDMFT can properly reproduce the experimental insulating state for all four compounds in the family without artificial tuning of compound-specific parameters. B3LYP still slightly underestimates the experimental peak positions for PES and IPES and the insulating gap for MnO and CoO. eDMFT slightly underestimates the PES/IPES gap in NiO but overall agrees with the experimental PES/IPES peak positions very well. When comparing with available ARPES, eDMFT compares much better than B3LYP. Many additional high-energy bands are observed in B3LYP that are not present either in eDMFT or identified in the experiment. Computed optical absorptions in $\mathrm{NiO}$ and $\mathrm{MnO}$ also show better agreement in peak positions and in the total intensity for the absorption coefficient between experiment and eDMFT than B3LYP, but B3LYP performs better in CoO. As the optical measurements are not recent, we caution that this conclusion might need revision if the optical measurement is redone in $\mathrm{CoO}$.

The failure of $\mathrm{GW}_{0}$ for these correlated systems is not surprising and were argued in the literature to be either due to lack of a proper starting point for computing dielectric function or due to the lack of self-consistency in electron screening. . $^{1,61,64}$ The inclusion of Hubbard $U$ in the starting point (applying $\mathrm{GW}_{0}$ on top of $\mathrm{LDA}+\mathrm{U}$ ) also gives an insulating solution for $\mathrm{FeO}$ and $\mathrm{CoO},{ }^{31,61,64}$ but this again requires fine-tuning of $U$, like in $\mathrm{GGA}+\mathrm{U}$, and is not attempted here.

The good performance of eDMFT is due to the inclusion of higher-order Feynman diagrams by the impurity solver, which allows one to compute the local correlation exactly. It also includes the electron scattering leading to a finite lifetime, which is beyond the limit of hybrid functional approaches, which only correct the exchange part of the interaction. In eDMFT, the $s$ and $p$ orbitals are treated at the DFT level for computational efficiency, to avoid the exponential scaling of the method with the number of interacting correlated orbitals. Full structural relaxation, which is routinely performed within DFT pseudopotential methods, is challenging for any all-electron linear augmented plane wave (LAPW)-based DFT and consequently also for beyond-DFT methods. Nevertheless, the calculation of forces (but not stresses) has recently become available in eDMFT. ${ }^{65}$

The beyond-DFT methods are computationally expensive, and we show in Table 1 the approximate cpu time and wall time for the typical self-consistent calculations in this class of materials, both of which are important for their widespread adoption. The efficiency of the parallelization can make the wall time very different from the corresponding cpu time, as the scaling of the particular method varies considerably between these methods.
Table 1. Approximate average computational cost per compound in various beyond-DFT approaches.

\begin{tabular}{llll}
\hline Approach & \# cores & Wall time $(\mathrm{h})$ & cpu-hours \\
\hline $\mathrm{mBJ}$ & 10 & 0.04 & 0.4 \\
GW & 160 & 1.8 & 300 \\
B3LYP & 48 & 32 & 2200 \\
eDMFT & 350 & 5.7 & 2000 \\
\hline
\end{tabular}

For example, the wall time of eDMFT calculation remains nearly inversely proportional to the number of available cpus up to approximately 100,000 cpu cores, as the computationally expensive part is based on the linearly parallelizable quantum Monte Carlo method. However, for more complex materials with larger unit cells, the scaling with computational resources is very different. The DFT part of the algorithm scales as $N^{3}$ while the eDMFT part scales as $N$, where $N$ is the number of correlated atoms in the unit cell, so that the slow Monte Carlo part of the algorithm may no longer dominate for very large unit cell calculations. In this case, the difference in cpu time between DFT and DFT+eDMFT would get substantially reduced. Owing to good parallelization, both the $\mathrm{GW}_{0}$ and eDMFT can be performed with short wall time, as compared to B3LYP. We note, however, that with an increasing number $N$ of atoms in the unit cell, the computational cost of B3LYP and $\mathrm{GW}_{0}{ }^{66}$ grows approximately as $N^{4}$, while the cost of eDMFT grows as $a N+b N^{3}$, with $b$ corresponding to the small DFT time. However, the constant $a$ in eDMFT depends on the temperature and scales approximately as $1 / T$. Finally, in terms of the total computational cost as well as the wall time required, the most and least expensive approaches in our study are B3LYP and $\mathrm{mBJ}$, respectively.

We expect that our results for the AFM phase of insulating binary TMOs should be representative of a broader class of moderately correlated materials with open $3 d$ shells and wellformed local moments. In particular, we predict that, for such materials, the performance of eDMFT is likely to be the best among the methods discussed here. We hope to expand our database to investigate the performance of these methods for a much wider range of materials.

In conclusion, we have proposed a new paradigm in which a wide range of DFT and beyond-DFT methods are applied to a 
selected set of target materials in a systematic and uniform manner in order to develop a systematic way of choosing the most accurate and efficient method for any given material. As a first demonstration, we have applied the GGA, GGA+U, mBJ, GW B3LYP, and DFT+eDMFT methods to a set of four prototypical binary TMOs, $\mathrm{MnO}, \mathrm{NiO}, \mathrm{FeO}$, and $\mathrm{CoO}$, in the AFM phase, and evaluated their performance for the DOS, spectral functions, and optical conductivity.

For these materials, we find that eDMFT is a preferred methodology that can reasonably well reproduce the ARPES, PES/IPES, and optics experiments without any material-specific tuning of parameters. B3LYP also performs well in reproducing the main features of the DOS but has issues in describing the ARPES and, in some of the TMOs, the optics as well.

Although we have studied only four compounds here, we can predict that, for moderately correlated AFM materials with open $3 d$ shells and well-described local moments, the performance of eDMFT is likely to be superior among the methods discussed here. To establish similar conclusions for other material classes, calculations are currently underway on a much broader range of materials. As we populate an open-source database containing the results, our findings show promise for accelerating the progress of computational material discovery and design, especially as applied to correlated materials. Our work is thus representative of recent trends toward the integration of fundamental physical theories and computational methodologies with database-driven science and engineering.

\section{METHODS}

Computational details

In this work, we use the full-potential LAPW method as described in the WIEN $2 \mathrm{k}^{67}$ software for various DFT and beyond-DFT methods, such as the $\mathrm{mBJ}$ potential $^{50}$ for meta-GGA, B3LYP ${ }^{15,68}$ for hybrid functionals, all electron GW (FHI-gap software ${ }^{51}$ ) for Hedin's GW formalism, and eDMFT $^{53,54}$ method for DMFT. For $\mathrm{mBJ}$ and B3LYP, we construct the initial wavefunction and eigenvalues with PBE functional in the GGA. For DFT, $\mathrm{DFT}+\mathrm{U}, \mathrm{mBJ}$, and B3LYP, we use $20 \times 20 \times 20 k$-points and $0.01 \mathrm{Ry}$ Gaussian broadening for computing DOS.

\section{$\mathrm{GGA}+\mathrm{U}$}

While the linear response theory tends to give smaller values of $U$ and usually quite accurately predicts the energetic and structural properties, the same $U$ is usually too small for a proper description of the spectrum..$^{31,69-71}$ The constrained-DFT gives larger values of $U$, which are often too large when compared with the experiment. This is because DFT $+\mathrm{U}$ solves the impurity problem within the Hartree-Fock method, and hence all the higher-order Feynman diagrams (beyond the exchange) should be accounted for in the method that computes the effective $U$. The $\mathrm{U}$ values in $\mathrm{GGA}+\mathrm{U}$ are $6.04,7.05,5.91$, and $6.88 \mathrm{eV}$ for $\mathrm{MnO}, \mathrm{NiO}, \mathrm{FeO}$, and $\mathrm{CoO}$, respectively, and obtained from ref. ${ }^{15}$.

\section{DFT+DMFT}

In eDMFT method, ${ }^{53,54}$ we use the LDA functional and the LAPW basis set as implemented in WIEN2k. ${ }^{67}$ The continuous time quantum Monte Carlo method $^{72}$ is used to solve the quantum impurity problem that is embedded within the Dyson equation for the solid, to obtain the local self-energy for the TM $d$ orbitals. The self-energy is then analytically continued with the maximum entropy method from the imaginary to the real axis, continuing the local cumulant function, to obtain the partial DOS. In eDMFT, where all such higher-order Feynman diagrams are explicitly calculated by the impurity solver, the amount of screening by the degrees of freedom not included in the method is substantially reduced, and the values of $U$ are larger and are quite successfully predicted by the selfconsistent constrained method. A fine $k$-point mesh of at least $10 \times 10 \times$ 10 -points in Monkhorst-Pack k-point grid and a total 100 million Monte Carlo steps for each iteration are used for the AFM phase of the TMO at T $=300 \mathrm{~K}$. To avoid tuning parameters, the Coulomb interaction $U$ and Hund's coupling $J_{H}$ are fixed at 10.0 and $1.0 \mathrm{eV}$, respectively, for all four TMOs. These values are computed by the constrained-eDMFT method. We use exact double counting between LDA and $\mathrm{DMFT}^{73}$ and we also compare our results with the fully localized limit (FLL) double counting ${ }^{74}$ scheme.

\section{GW}

We perform single-shot GW and $\mathrm{GW}_{0}$ using the FHI-gap software package $^{51}$ where GW self-energy is computed within the all-electron LAPW basis of WIEN2K. We use $4 \times 4 \times 4 k$-point grids and include unoccupied bands with energy up to $50 \mathrm{Ry}$. We also include high-energy local orbitals in the $\mathrm{GW}_{0}$ calculations. About $1000 \mathrm{k}$-points are considered for computing the DOS, where we first compute the quasiparticle energies in a sparse $k$-mesh and then interpolate to a much finer $k$-mesh. The muffin tin radii (in Bohr) for $\mathrm{Mn}$ and $\mathrm{O}$ atoms are $(2.10,1.77)$ for $\mathrm{MnO}$; $(2.05,1.75)$ for $\mathrm{FeO}$; and $(1.97,1.75)$ for $\mathrm{CoO}$ and $\mathrm{NiO} .{ }^{31}$ The Gaussian broadening and $k$-point sampling for computing DOS are kept at least $\sim 0.01$ Ry and $10 \times$ $10 \times 10$, respectively. Similar values were used in the ref. ${ }^{31}$.

\section{Optical absorption computations}

Within eDMFT, we obtain the imaginary part of the dielectric function from the real part of the optical conductivity and then perform the Kramers-Kronig operations to compute the absorption coefficients.

\section{DATA AVAILABILITY}

The data that support the findings of this study are available from NIST-JARVIS website (https://www.ctcms.nist.gov/knc6/JVASP.html).

\section{CODE AVAILABILITY}

We have used the WIEN2K, ${ }^{67}$ DFT+eDMFT, and FHI-GW codes to generate the data. WIEN2K is a commercially available software package. DFT+eDMFT and FHI-GW are freely available and can be obtained from http://hauleweb.rutgers.edu/tutorials and ref. ${ }^{51}$, respectively.

Received: 23 July 2019; Accepted: 5 November 2019; Published online: 29 November 2019

\section{REFERENCES}

1. Kotliar, G. \& Vollhardt, D. Strongly correlated materials: insights from dynamical mean-field theory. Phys. Today 57, 53-59 (2004).

2. Onida, G., Reining, L. \& Rubio, A. Electronic excitations: density-functional versus many-body Green's-function approaches. Rev. Mod. Phys. 74, 601 (2002).

3. Georges, A., Kotliar, G., Krauth, W. \& Rozenberg, M. J. Dynamical mean-field theory of strongly correlated fermion systems and the limit of infinite dimensions. Rev. Mod. Phys. 68, 13-125 (1996).

4. Kotliar, G. et al. Electronic structure calculations with dynamical mean-field theory. Rev. Mod. Phys. 78, 865-951 (2006).

5. Choudhary, K. et al. Computational screening of high-performance optoelectronic materials using OptB88vdW and Tb-mBJ formalisms. Sci. Data 5, 180082 (2018).

6. Stevanović, V., Lany, S., Zhang, X. \& Zunger, A. Correcting density functional theory for accurate predictions of compound enthalpies of formation: fitted elemental-phase reference energies. Phys. Rev. B 85, 115104 (2012).

7. Li, W., Walther, C. F. J., Kuc, A. \& Heine, T. Density functional theory and beyond for band-gap screening: performance for transition-metal oxides and dichalcogenides. J. Chem. Theory Comput. 9, 2950-2958 (2013).

8. Lanatà, N., Lee, T.-H., Yao, Y.-X., Stevanović, V. \& Dobrosavljević, V. Connection between Mott physics and crystal structure in a series of transition metal binary compounds. npj Comput. Mater. 5, 30 (2019).

9. Perdew, J. P. \& Zunger, A. Self-interaction correction to density-functional approximations for many-electron systems. Phys. Rev. B 23, 5048-5079 (1981).

10. Jones, R. O. \& Gunnarsson, O. The density functional formalism, its applications and prospects. Rev. Mod. Phys. 61, 689-746 (1989).

11. Anisimov, V. I., Aryasetiawan, F. \& Lichtenstein, A. I. First-principles calculations of the electronic structure and spectra of strongly correlated systems: the LDA+U method. J. Phys. Conden. Matter 9, 767-808 (1997).

12. Himmetoglu, B., Floris, A., de Gironcoli, S. \& Cococcioni, M. Hubbard-corrected DFT energy functionals: the LDA $+U$ description of correlated systems. Int. J. Quant. Chem. 114, 14-49 (2013). 
13. Gopal, P. et al. Improved electronic structure and magnetic exchange interactions in transition metal oxides. J. Phys. Condens. Matter 29, 444003 (2017).

14. Payne, M. C., Teter, M. P., Allan, D. C., Arias, T. A. \& Joannopoulos, J. D. Iterative minimization techniques for ab initio total-energy calculations: molecular dynamics and conjugate gradients. Rev. Mod. Phys. 64, 1045-1097 (1992).

15. Tran, F., Blaha, P., Schwarz, K. \& Novák, P. Hybrid exchange-correlation energy functionals for strongly correlated electrons: applications to transition-metal monoxides. Phys. Rev. B 74, 155108 (2006).

16. Heyd, J., Scuseria, G. E. \& Ernzerhof, M. Hybrid functionals based on a screened Coulomb potential. J. Chem. Phys. 118, 8207-8215 (2003).

17. Perdew, J. P., Ernzerhof, M. \& Burke, K. Rationale for mixing exact exchange with density functional approximations. J. Chem. Phys. 105, 9982-9985 (1996).

18. Lee, C., Yang, W. \& Parr, R. G. Development of the Colle-Salvetti correlationenergy formula into a functional of the electron density. Phys. Rev. B 37, 785-789 (1988).

19. Hedin, L. New method for calculating the one-particle Green's function with application to the electron-gas problem. Phys. Rev. 139, A796 (1965).

20. Hybertsen, M. S. \& Louie, S. G. Electron correlation in semiconductors and insulators: band gaps and quasiparticle energies. Phys. Rev. B 34, 5390-5413 (1986).

21. Aryasetiawan, F. \& Gunnarsson, O. The GW method. Rep. Progr. Phys. 61, 237-312 (1998).

22. Yin, Z. P., Haule, K. \& Kotliar, G. Kinetic frustration and the nature of the magnetic and paramagnetic states in iron pnictides and iron chalcogenides. Nat. Mater. 10, 932 (2011)

23. Mandal, S., Zhang, P., Ismail-Beigi, S. \& Haule, K. How correlated is the FeSe/SrTiO system?. Phys. Rev. Lett. 119, 067004 (2017).

24. Liu, M. et al. Nature of magnetic excitations in superconducting $\mathrm{BaFe}_{1.9} \mathrm{Ni}_{0.1} \mathrm{As}_{2}$. Nat. Phys. 8, 376-381 (2012).

25. Mandal, S., Cohen, R. E. \& Haule, K. Pressure suppression of electron correlation in the collapsed tetragonal phase of $\mathrm{CaFe}_{2} \mathrm{As}_{2}$ : a DFT-DMFT investigation. Phys. Rev. B 90, 060501 (2014). (R).

26. Backes, S., Jeschke, H. O. \& Valentí, R. Microscopic nature of correlations in multiorbital $\mathrm{AFe}_{2} \mathrm{As}_{2}(\mathrm{~A}=\mathrm{K}, \mathrm{Rb}, \mathrm{Cs})$ : Hund's coupling versus coulomb repulsion. Phys. Rev. B 92, 195128 (2015).

27. Kuneš, J. et al. Collapse of magnetic moment drives the Mott transition in $\mathrm{MnO}$. Nat. Mat. 7, 198-202 (2008).

28. Shim, J. H., Haule, K. \& Kotliar, G. Fluctuating valence in a correlated solid and the anomalous properties of [dgr]-plutonium. Nature 446, 513-516 (2007).

29. Haule, K., Birol, T. \& Kotliar, G. Covalency in transition-metal oxides within allelectron dynamical mean-field theory. Phys. Rev. B 90, 075136 (2014).

30. Kent, P. R. C. \& Kotliar, G. Toward a predictive theory of correlated materials. Science 361, 348-354 (2018).

31. Jiang, H., Gomez-Abal, R. I., Rinke, P. \& Scheffler, M. First-principles modeling of localized $d$ states with the $d$ approach. Phys. Rev. B 82, 045108 (2010).

32. Yin, Q., Gordienko, A., Wan, X. \& Savrasov, S. Y. Calculated momentum dependence of Zhang-Rice states in transition metal oxides. Phys. Rev. Lett. 100, 066406 (2008).

33. Hariki, A., Uozumi, T. \& Kuneš, J. LDA+DMFT approach to core-level spectroscopy: application to $3 d$ transition metal compounds. Phys. Rev. B 96, 045111 (2017).

34. Seo, D.-H., Urban, A. \& Ceder, G. Calibrating transition-metal energy levels and oxygen bands in first-principles calculations: accurate prediction of redox potentials and charge transfer in lithium transition-metal oxides. Phys. Rev. B 92, 115118 (2015).

35. Takahashi, M. \& Igarashi, J.-i Local approach to electronic excitations in $\mathrm{MnO}, \mathrm{FeO}$, CoO, and NiO. Phys. Rev. B 54, 13566-13574 (1996).

36. Cohen, R. E., Mazin, I. I. \& Isaak, D. G. Magnetic collapse in transition metal oxides at high pressure: implications for the earth. Science 275, 654-657 (1997).

37. Ohta, K. et al. Experimental and theoretical evidence for pressure-induced metallization in $\mathrm{FeO}$ with rocksalt-type structure. Phys. Rev. Lett. 108, 026403 (2012).

38. Kuneš, J., Anisimov, V. I., Skornyakov, S. L., Lukoyanov, A. V. \& Vollhardt, D. NiO: correlated band structure of a charge-transfer insulator. Phys. Rev. Lett. 99, 156404 (2007).

39. Shen, Z.-X. et al. Electronic structure of NiO: correlation and band effects. Phys. Rev. B 44, 3604-3626 (1991).

40. van Elp, J., Potze, R. H., Eskes, H., Berger, R. \& Sawatzky, G. A. Electronic structure of MnO. Phys. Rev. B 44, 1530-1537 (1991).

41. Sawatzky, G. A. \& Allen, J. W. Magnitude and origin of the band gap in NiO. Phys. Rev. Lett. 53, 2339-2342 (1984).

42. van Elp, J. et al. Electronic structure of $\mathrm{CoO}$, Li-doped $\mathrm{CoO}$, and $\mathrm{LiCO}_{2}$. Phys. Rev. B 44, 6090-6103 (1991)

43. Zimmermann, R. et al. Electronic structure of 3d-transition-metal oxides: on-site Coulomb repulsion versus covalency. J. Phys. Condens. Matter 11, 1657-1682 (1999).
44. Terakura, K., Oguchi, T., Williams, A. R. \& Kübler, J. Band theory of insulating transition-metal monoxides: band-structure calculations. Phys. Rev. B 30, 4734-4747 (1984).

45. Zaanen, J., Sawatzky, G. A. \& Allen, J. W. Band gaps and electronic structure of transition-metal compounds. Phys. Rev. Lett. 55, 418-421 (1985).

46. Roth, W. L. Magnetic structures of $\mathrm{MnO}, \mathrm{FeO}, \mathrm{CoO}$, and NiO. Phys. Rev. 110 1333-1341 (1958).

47. Perdew, J. P., Burke, K. \& Ernzerhof, M. Generalized gradient approximation made simple. Phys. Rev. Lett. 77, 3865 (1996).

48. Anisimov, V. I., Solovyev, I. V., Korotin, M. A., Czyżyk, M. T. \& Sawatzky, G. A Density-functional theory and $\mathrm{NiO}$ photoemission spectra. Phys. Rev. B 48 16929-16934 (1993).

49. Liechtenstein, A. I., Anisimov, V. I. \& Zaanen, J. Density-functional theory and strong interactions: orbital ordering in Mott-Hubbard insulators. Phys. Rev. B 52 R5467-R5470 (1995).

50. Tran, F. \& Blaha, P. Accurate band gaps of semiconductors and insulators with a semilocal exchange-correlation potential. Phys. Rev. Lett. 102, 226401 (2009).

51. Jiang, $\mathrm{H}$. et al. FHI-gap: a GW code based on the all-electron augmented plane wave method. Comput. Phys. Commun. 184, 348-366 (2013).

52. Becke, A. D. A new mixing of Hartree-Fock and local density-functional theories. J. Chem. Phys. 98, 1372 (1993).

53. Haule, K., Yee, C.-H. \& Kim, K. Dynamical mean-field theory within the fullpotential methods: electronic structure of Ce-115 materials. Phys. Rev. B 81 195107 (2010).

54. Haule, K. Structural predictions for correlated electron materials using the functional dynamical mean field theory approach. J. Phys. Soc. Jpn. 87, 041005 (2018).

55. Wyckoff, R. W. G. Crystal Structures (Interscience, New York, 1964).

56. McCammon, C. A. \& Liu, L.-g The effects of pressure and temperature on nonstoichiometric wüstite, $\mathrm{Fe}_{\mathrm{x}} \mathrm{O}$ : the iron-rich phase boundary. Phys. Chem. Miner. 10, 106-113 (1984).

57. Carey, M., Spada, F., Berkowitz, A., Cao, W. \& Thomas, G. Preparation and structural characterization of sputtered $\mathrm{CoO}, \mathrm{NiO}$, and $\mathrm{Ni}_{0.5} \mathrm{Co}_{0.5} \mathrm{O}$ thin epitaxial films. $\mathrm{J}$. Mater. Res. 6, 2680-2687 (1991).

58. Bartel, L. C. \& Morosin, B. Exchange striction in NiO. Phys. Rev. B 3, 1039-1043 (1971).

59. Mandal, S., Haule, K., Rabe, K. M. \& Vanderbilt D. Influence of magnetic ordering on the spectral properties of binary transition metal oxides. arXiv:1909.09189.

60. Janowski, J., Nowotny, J. \& Rekas, M. in Non-Stoichiometry and Defect Structure of $\mathrm{FeO}$ (eds Nowotny J. \& Weppner W.) 115-121 (Springer, Dordrecht, 1989).

61. Rödl, C. \& Bechstedt, F. Optical and energy-loss spectra of the antiferromagnetic transition metal oxides $\mathrm{MnO}, \mathrm{FeO}, \mathrm{CoO}$, and $\mathrm{NiO}$ including quasiparticle and excitonic effects. Phys. Rev. B 86, 235122 (2012).

62. Drokin, N. A., Ovchinnikov, S. G. \& Ryabinkina, L. I. Photoconductivity of alphaMnS and MnO. Fiz. Tverd. Tela (Leningrad) 18, 173 (1976)

63. Powell, R. J. \& Spicer, W. E. Optical properties of $\mathrm{NiO}$ and CoO. Phys. Rev. B 2, 2182-2193 (1970).

64. Rödl, C., Fuchs, F., Furthmüller, J. \& Bechstedt, F. Quasiparticle band structures of the antiferromagnetic transition-metal oxides $\mathrm{MnO}, \mathrm{FeO}, \mathrm{CoO}$, and $\mathrm{NiO}$. Phys. Rev. B 79, 235114 (2009).

65. Haule, K. \& Pascut, G. L. Forces for structural optimizations in correlated materials within a DFT+embedded DMFT functional approach. Phys. Rev. B 94, 195146 (2016).

66. Kim, M. et al. Scalable GW software for quasiparticle properties using OpenAtom. Comput. Phys. Commun. 244, 427-441 (2019).

67. Blaha, P., Schwarz, K., Madsen, G., Kvasnicka, D. \& Luitz, J. WIEN2k: An Augmented Plane Wave Plus Local Orbitals Program for Calculating Crystal Properties (Vienna University of Technology, Austria, 2001).

68. Becke, A. D. Density-functional thermochemistry. III. The role of exact exchange. J. Chem. Phys. 98, 5648-5652 (1993).

69. Anisimov, V. I., Zaanen, J. \& Andersen, O. K. Band theory and mott insulators: Hubbard U instead of Stoner I. Phys. Rev. B 44, 943-954 (1991).

70. Pickett, W. E, Erwin, S. C. \& Ethridge, E. C. Reformulation of the LDA + $u$ method for a local-orbital basis. Phys. Rev. B. 58, 1201-1209 (1998).

71. Cococcioni, M. \& de Gironcoli, S. Linear response approach to the calculation of the effective interaction parameters in the LDA $+U$ method. Phys. Rev. B 71 035105 (2005).

72. Haule, K. Quantum Monte Carlo impurity solver for cluster dynamical mean-field theory and electronic structure calculations with adjustable cluster base. Phys. Rev. B 75, 155113 (2007)

73. Haule, K. Exact double counting in combining the dynamical mean field theory and the density functional theory. Phys. Rev. Lett. 115, 196403 (2015).

74. Czyżyk, M. T. \& Sawatzky, G. A. Local-density functional and on-site correlations: the electronic structure of $\mathrm{La}_{2} \mathrm{CuO}_{4}$ and $\mathrm{La}_{2} \mathrm{CuO}_{3}$. Phys. Rev. B 49, 14211-14228 (1994) 
75. Villalobos, J. J., Parashar, M., Rodero, I. \& Brennan-Tonetta, M. High performance computing at the Rutgers Discovery Informatics Institute. Technical Report (2018).

\section{ACKNOWLEDGEMENTS}

We thank Steven Louie, Hong Jiang, and Zhenglu Li for helpful discussions related to GW calculations. We thank G. L. Pascut for helpful discussions. We are grateful to NIST for hosting our database. We thank Kamal Choudhary and Francesca Tavazza for their help in hosting our data in the NIST-JARVIS database. The computations were performed at the Extreme Science and Engineering Discovery Environment (XSEDE), which is supported by National Science Foundation grant number ACl-1548562, Rutgers HPC (RUPC). This research also used resources from the Rutgers Discovery Informatics Institute ${ }^{75}$ which are supported by Rutgers and the State of New Jersey. This research was funded by NSF DMREF DMR-1629059 and NSF DMREF DMR-1629346.

\section{AUTHOR CONTRIBUTIONS}

S.M. carried out the calculations. All authors discussed the results and co-wrote the paper.

\section{COMPETING INTERESTS}

The authors declare no competing interests.

\section{ADDITIONAL INFORMATION}

Correspondence and requests for materials should be addressed to S.M.

Reprints and permission information is available at http://www.nature.com/ reprints

Publisher's note Springer Nature remains neutral with regard to jurisdictional claims in published maps and institutional affiliations.

\section{(c) (i)}

Open Access This article is licensed under a Creative Commons Attribution 4.0 International License, which permits use, sharing, adaptation, distribution and reproduction in any medium or format, as long as you give appropriate credit to the original author(s) and the source, provide a link to the Creative Commons license, and indicate if changes were made. The images or other third party material in this article are included in the article's Creative Commons license, unless indicated otherwise in a credit line to the material. If material is not included in the article's Creative Commons license and your intended use is not permitted by statutory regulation or exceeds the permitted use, you will need to obtain permission directly from the copyright holder. To view a copy of this license, visit http://creativecommons. org/licenses/by/4.0/.

(c) The Author(s) 2019 